\title{
On Parameterised Quadratic Inverse Eigenvalue Problem
}

\author{
Meiling Xiang and Hua Dai* \\ Department of Mathematics, Nanjing University of Aeronautics and Astronautics, \\ Nanjing 210016, China.
}

Received 25 March 2021; Accepted (in revised version) 23 August 2021.

\begin{abstract}
It is shown that if prescribed eigenvalues are distinct, then the parameterised quadratic inverse eigenvalue problem is equivalent to a multiparameter eigenvalue problem. Moreover, a sufficient condition for the problem solvability is established. In order to find approximate solution of this problem, we employ the Newton method based on the smooth $Q R$-decomposition with column pivoting and prove its locally quadratic convergence. Numerical examples illustrate the effectiveness of the method.
\end{abstract}

AMS subject classifications: $65 \mathrm{~F} 15$

Key words: Quadratic inverse eigenvalue problem, multiparameter eigenvalue problem, smooth $Q R$-decomposition, Newton method.

\section{Introduction}

There is a wide class of problems, which can be reduced to the system of second-order differential equations

$$
M \ddot{x}(t)+C \dot{x}(t)+K x(t)=f(t),
$$

where $M, C, K \in \mathbb{R}^{n \times n}$, det $M \neq 0$ are respectively the analytical mass, damping, and stiffness matrices and $x(t), f(t) \in \mathbb{R}^{n}$ the displacement and external force vectors depending on time $t$. Physical and geometric parameters characterising the underlying physical system are embedded in the coefficient matrices $M, C$ and $K$. From a priori known physical and geometric parameters such as mass, damping coefficient, elasticity, length, area and so on, the process of analysing and deriving the dynamical behavior of the system is referred to as the direct problem. It is well known that if $x(t)=e^{\lambda t} v, \lambda \in \mathbb{C}, v \in \mathbb{C}^{n}$ a fundamental solution to (1.1), then $\lambda$ and $v$ satisfy the quadratic eigenvalue problem (QEP)

$$
Q(\lambda) v=0,
$$

*Corresponding author. Email addresses: xi angmeiling@nuaa.edu.cn (M.L. Xiang), hdai@nuaa.edu.cn (H. Dai) 
where $Q(\lambda)=\lambda^{2} M+\lambda C+K$ and is called the quadratic pencil. Since $M$ is a nonsingular matrix, the dynamical behavior of the system (1.1) may be interpreted via $2 n$ eigenvalues $\left\{\lambda_{i}\right\}_{i=1}^{2 n}$ and eigenvectors $\left\{v_{i}\right\}_{i=1}^{2 n}$ of the QEP (1.2). Various quadratic eigenvalue problems have been studied so far and we refer the readers to [31] for a survey on their applications, mathematical properties, and corresponding numerical methods.

On the other hand, the inverse problem is to determine physical and geometric parameters for three matrices $M, C, K$ such that the corresponding system has a prescribed dynamical behavior. More exactly, we consider the following problem.

Problem 1.1 (Parameterised Quadratic Inverse Eigenvalue Problem (PQIEP)). Let $M \in$ $\mathbb{R}^{n \times n}$ be a non-singular matrix and $C_{i}, K_{i} \in \mathbb{R}^{n \times n}, i=0,1, \ldots, 2 n$. For any distinct complex numbers $\lambda_{1}, \lambda_{2}, \ldots, \lambda_{2 n}$, find a vector $c=\left(c_{1}, c_{2}, \ldots, c_{2 n}\right)^{T} \in \mathbb{R}^{2 n}$ or $\mathbb{C}^{2 n}$ such that the quadratic eigenvalue problem

$$
\left[\lambda^{2} M+\lambda C(c)+K(c)\right] x=0
$$

with the matrices

$$
C(c):=C_{0}+\sum_{i=1}^{2 n} c_{i} C_{i}, K(c):=K_{0}+\sum_{i=1}^{2 n} c_{i} K_{i}
$$

has the eigenvalues $\lambda_{1}, \lambda_{2}, \ldots, \lambda_{2 n}$.

Such problems appear in various practical applications including structural design [22] and finite element model updating [7,8]. The general theory, numerical methods and applications of the standard inverse eigenvalue problem - i.e. if $M=0$ and $C(c)$ is the identity matrix, are discussed in $[6,18,33,34]$. Mathematical theory and algorithms of the parameterised generalized inverse eigenvalue problem - i.e. if $M=0$, are studied in $[9,11,12,22,29]$.

Using the determinant evaluations proposed by Lancaster [24] and Biegler-König [3] and further developed by Friedland et al. [18], Elhay and Ram [16] reformulated PQIEP as the system of nonlinear equations

$$
F(c)=\left(\begin{array}{c}
\operatorname{det}\left(\lambda_{1}^{2} M+\lambda_{1} C(c)+K(c)\right) \\
\operatorname{det}\left(\lambda_{2}^{2} M+\lambda_{2} C(c)+K(c)\right) \\
\vdots \\
\operatorname{det}\left(\lambda_{2 n}^{2} M+\lambda_{2 n} C(c)+K(c)\right)
\end{array}\right)
$$

and proposed a Newton method for its solution. However, this method may suffer from ill-conditioning [18] and is not computationally attractive.

Assume that Problem 1.1 has a solution $c^{*}$. Then there is a neighborhood of $c^{*}$ such that the eigenvalues $\lambda_{i}(c)$ of the quadratic pencil $Q_{c}(\lambda)=\lambda^{2} M+\lambda C(c)+K(c)$ are distinct differentiable functions [1]. In this neighborhood, Elhay and Ram [17] considered the 
system of nonlinear equations

$$
F(c)=\left(\begin{array}{c}
\lambda_{1}(c)-\lambda_{1} \\
\lambda_{2}(c)-\lambda_{2} \\
\vdots \\
\lambda_{2 n}(c)-\lambda_{2 n}
\end{array}\right) .
$$

This is the most natural formulation, so that it was used by many authors to study additive and multiplicative inverse eigenvalue problems $[15,20]$, standard inverse eigenvalue problem $[4,5,18,28,30,32]$ and generalised inverse eigenvalue problem [12]. In order to efficiently apply a Newton method to the system (1.5), it is important to reorder the eigenvalues in a suitable way. However, since in general the eigenvalues of the quadratic pencil $Q_{c}(\lambda)$ are complex numbers, the ordering of the eigenvalues is a nontrivial task. Assuming that the number of real and complex eigenvalues in each iteration remains the same as the number of prescribed real and complex eigenvalues, Elhay and Ram [17] developed a Newton method for solving PQIEP by finding a root of the nonlinear system (1.5). But the problem of the eigenvalues ordering was not addressed without such an assumption. Using the Hungarian method [23], Datta and Sokolov [14] solved the matching problem of the eigenvalues, and presented a Newton method, an alternating projection method and a hybrid method combined the Newton method with the alternating projection method for PQIEP. However, at every iteration step each of the methods mentioned computes all eigenvalues and eigenvectors of the problem (1.5) and the eigenvalues have to be reordered by the Hungarian method, which is very time-consuming operations.

One of the important issues to study, concerns the solvability of PQIEP, and up to now there are no sufficient solvability conditions for this problem. Motivated by the ideas of $[11,21]$, we consider the solvability of PQIEP, and transform PQIEP into a multiparameter eigenvalue problem under the condition that the prescribed eigenvalues are distinct. Consequent application of results from the theory of multiparameter eigenvalue problems allows us to obtain a sufficient condition of solvability of PQIEP.

Using the smooth $Q R$-decomposition with column pivoting [25], Li [26] proposed a numerical method for the standard inverse eigenvalue problem. Besides, the $Q R$-like decomposition with column pivoting [13], was exploited in Dai [9] in an algorithm for solving the parameterised generalised inverse eigenvalue problem. In order to avoid the eigenvalue computing and reordering, we develop a numerical algorithm for PQIEP, which is based on the smooth $Q R$-decomposition.

This paper is organised as follows. In Section 2, PQIEP is reduced to an equivalent multiparameter eigenvalue problem and a sufficient solvability condition for PQIEP is presented. In Section 3, the Newton method employing the smooth $Q R$-decomposition with column pivoting is applied to PQIEP and its locally quadratic convergence is shown. Section 4 contains numerical examples, which show the effectiveness of the method. Finally, in Section 5 a few conclusions are drawn.

Let us explain the notations we use. It is clear from the previous discussion that $\mathbb{R}^{m \times n}$ and $\mathbb{C}^{m \times n}$ refer to the sets of real and complex $m \times n$ matrices, respectively. In particular, 
$\mathbb{R}^{m}=\mathbb{R}^{m \times 1}, \mathbb{C}^{m}=\mathbb{C}^{m \times 1}, \mathbb{R}=\mathbb{R}^{1}$ and $\mathbb{C}=\mathbb{C}^{1}$. Besides, $\mathscr{U}_{n}$ and $\mathscr{R}_{n}$ stand for the sets of all $n \times n$ unitary matrices and all $n \times n$ upper-triangular matrices, respectively. $O$ and $I$ denote the zero matrix and identity matrix of suitable size, $e_{j}$ is the $j$-th column vector of $I, I^{(j)}=\left[e_{1}, e_{2}, \ldots, e_{j}\right],\|\cdot\|_{2}$ represents the Euclidean vector norm or the induced matrix norm, and $\|\cdot\|_{F}$ denotes the Frobenius matrix norm. If $A$ is an $m \times n$ matrix, then $A^{T}, A^{H}$, $\operatorname{rank}(\mathrm{A})$ and $\operatorname{ker}(A)$ are respectively the transpose, conjugate transpose, rank and kernel of $A$. The direct sum of real or complex finite-dimensional Hilbert spaces is denoted by $\oplus$ and $\otimes$ is the tensor product of vectors or matrices. Throughout this paper, we always assume that the given eigenvalues $\lambda_{1}, \lambda_{2}, \ldots, \lambda_{2 n}$ are distinct and the solution to PQIEP is written as $c^{*}$. To ensure that the number of free parameters does not degenerate, we also assume that $2 n$ matrices $\left[C_{j}, K_{j}\right]$ are linearly independent in the space of $n \times 2 n$ matrices.

\section{A Sufficient Condition of Solvability}

Let briefly recall some facts from the theory of multiparameter eigenvalue problem cf. [2]. Let $A_{i, j} \in \mathbb{R}^{n_{i} \times n_{i}}$ or $\mathbb{C}^{n_{i} \times n_{i}}, i=1,2, \ldots, m, j=0,1, \ldots, m$. The multiparameter eigenvalue problem is to find scalars $\mu_{0}, \mu_{1}, \ldots, \mu_{m}$, not all of them zeros, and nonzero vectors $x_{i} \in \mathbb{C}^{n_{i}}, i=1,2, \ldots, m$ such that

$$
\begin{gathered}
\left(\mu_{0} A_{1,0}+\mu_{1} A_{1,1}+\cdots+\mu_{m} A_{1, m}\right) x_{1}=0, \\
\left(\mu_{0} A_{2,0}+\mu_{1} A_{2,1}+\cdots+\mu_{m} A_{2, m}\right) x_{2}=0, \\
\cdots \cdots \\
\left(\mu_{0} A_{m, 0}+\mu_{1} A_{m, 1}+\cdots+\mu_{m} A_{m, m}\right) x_{m}=0 .
\end{gathered}
$$

The $(m+1)$-tuple $\mu=\left(\mu_{0}, \mu_{1}, \ldots, \mu_{m}\right)$ and the vector $x=x_{1} \otimes x_{2} \otimes \cdots \otimes x_{m}$ are called the eigenvalue and the corresponding eigenvector of the problem (2.1), respectively. It is clear that $\mu=\left(\mu_{0}, \mu_{1}, \ldots, \mu_{m}\right)$ is an eigenvalue of the problem (2.1) if and only if

$$
\operatorname{ker}\left(\sum_{j=0}^{m} \mu_{j} A_{i, j}\right) \neq\{0\}, \quad i=1,2, \ldots, m,
$$

or, equivalently, if and only if

$$
\operatorname{det}\left(\sum_{j=0}^{m} \mu_{j} A_{i, j}\right)=0, \quad i=1,2, \ldots, m .
$$

Let $\mathbf{H}_{i}$ denote the Hilbert space $\mathbb{R}^{n_{i}}$ or $\mathbb{C}^{n_{i}}$ and $\mathbf{H}=\mathbf{H}_{1} \otimes \mathbf{H}_{2} \otimes \cdots \otimes \mathbf{H}_{m}$ be the tensor product space. Then $A_{i, j}, i=1,2, \ldots, m, j=0,1, \ldots, m$ are linear operators on $\mathbf{H}_{i}$. For a decomposable tensor $x=x_{1} \otimes x_{2} \otimes \cdots \otimes x_{m} \in \mathbf{H}$, the induced operator $A_{i, j}^{\dagger}$ is defined by

$$
\begin{aligned}
& A_{i, j}^{\dagger}\left(x_{1} \otimes \cdots \otimes x_{m}\right) \\
= & x_{1} \otimes \cdots \otimes x_{i-1} \otimes A_{i, j} x_{i} \otimes x_{i+1} \otimes \cdots \otimes x_{m}, \quad i=1,2, \ldots, m, \quad j=0,1, \ldots, m .
\end{aligned}
$$


An important property of these induced operators is that they commute with each other i.e.

$$
A_{i, j}^{\dagger} A_{k, j}^{\dagger}=A_{k, j}^{\dagger} A_{i, j}^{\dagger}, \quad i \neq k, \quad i, k=1,2, \ldots, m, \quad j=0,1, \ldots, m .
$$

In order to reduce the multiparameter eigenvalue problem (2.1) to a joint eigenvalue problem, we define the operator-valued determinants by

$$
\Delta_{j}=(-1)^{j} \operatorname{det}\left(\begin{array}{ccccc}
A_{1,0}^{\dagger} & \cdots & \widehat{A}_{1, j}^{\dagger} & \cdots & A_{1, m}^{\dagger} \\
\vdots & & \vdots & & \vdots \\
A_{m, 0}^{\dagger} & \cdots & \widehat{A}_{m, j}^{\dagger} & \cdots & A_{m, m}^{\dagger}
\end{array}\right), \quad j=0,1, \ldots, m,
$$

where $\widehat{A}_{i, j}$ means the omission of the full array $A_{i, j}^{\dagger}$ obtained by deleting the $(j+1)$-th column, and the right-hand side of (2.3) can be expanded in the usual manner for determinants, in which the products of the entries are interpreted as the composites of the operators concerned.

It is shown in [2] that the multiparameter eigenvalue problem (2.1) is equivalent to the following joint eigenvalue problem:

$$
\Delta^{-1} \Delta_{j} x=\mu_{j} x, \quad j=0,1, \ldots, m,
$$

where $\Delta=\sum_{j=0}^{m} \gamma_{j} \Delta_{j}$ is assumed to be nonsingular for some scalars $\gamma_{0}, \gamma_{1}, \ldots, \gamma_{m}$.

Theorem 2.1 (cf. Atkinson [2]). If there are scalars $\gamma_{0}, \gamma_{1}, \ldots, \gamma_{m}$ such that $\Delta=\sum_{j=0}^{m} \gamma_{j} \Delta_{j}$ is nonsingular, then for an eigenvalue $\mu=\left(\mu_{0}, \mu_{1}, \ldots, \mu_{m}\right)$ of the problem (2.1), we have

$$
\sum_{j=0}^{m} \gamma_{j} \mu_{j} \neq 0
$$

Up to a scaling factor, the eigenvalues of the problem (2.1) are the simultaneous eigenvalues of the joint eigenvalue problem (2.4). Moreover, all operators $\Delta^{-1} \Delta_{j}, j=1,2, \ldots, m$ mutually commute - i.e.

$$
\Delta_{i} \Delta^{-1} \Delta_{j}=\Delta_{j} \Delta^{-1} \Delta_{i}, \quad i, j=1,2, \ldots, m .
$$

Now we consider the solvability of PQIEP. Suppose that it has a solution $c \in \mathbb{C}^{2 n}$, i.e. there are nonzero vectors $x_{i} \in \mathbb{C}^{n}$ such that

$$
\left[\lambda_{i}^{2} M+\lambda_{i} C(c)+K(c)\right] x_{i}=0, \quad i=1,2, \ldots, 2 n .
$$

Using the notations

$$
A_{i, 0}=\lambda_{i}^{2} M+\lambda_{i} C_{0}+K_{0}, \quad A_{i, j}=\lambda_{i} C_{j}+K_{j}, \quad i, j=1,2, \ldots, 2 n,
$$

we rewrite (2.5) in the form

$$
\begin{gathered}
\left(A_{1,0}+c_{1} A_{1,1}+\cdots+c_{2 n} A_{1,2 n}\right) x_{1}=0, \\
\left(A_{2,0}+c_{1} A_{2,1}+\cdots+c_{2 n} A_{2,2 n}\right) x_{2}=0, \\
\cdots \cdots \\
\left(A_{2 n, 0}+c_{1} A_{2 n, 1}+\cdots+c_{2 n} A_{2 n, 2 n}\right) x_{2 n}=0 .
\end{gathered}
$$


This means that $c \in \mathbb{C}^{2 n}$ is an eigenvalue of the multiparameter eigenvalue problem (2.7), and $x=x_{1} \otimes x_{2} \otimes \cdots \otimes x_{2 n}$ is the corresponding eigenvector. Conversely, if $c \in \mathbb{C}^{2 n}$ and $x=$ $x_{1} \otimes x_{2} \otimes \cdots \otimes x_{2 n}$ are the eigenvalue and corresponding eigenvector of the multiparameter eigenvalue problem (2.7), respectively, then $c \in \mathbb{C}^{2 n}$ is a solution of PQIEP. So we can obtain the following result.

Theorem 2.2. If the given eigenvalues $\lambda_{1}, \lambda_{2}, \ldots, \lambda_{2 n}$ are distinct, then $c=\left(c_{1}, c_{2}, \ldots, c_{2 n}\right)^{T}$ is a solution of PQIEP if and only if it is an eigenvalue of the multiparameter eigenvalue problem (2.7).

Combining the definition of $A_{i, j}^{\dagger}$ in (2.2) with the properties of the Kronecker product [10], we represent the induced operators $A_{i, j}^{\dagger}$ for $A_{i, j}$ in (2.6) in the form

$$
A_{i, j}^{\dagger}=I \otimes \cdots \otimes I \otimes A_{i, j} \otimes I \otimes \cdots \otimes I, \quad i=1,2, \ldots, 2 n, \quad j=0,1, \ldots, 2 n .
$$

For the system (2.7) we also consider the operator-valued determinants $T_{0}, T_{1}, \ldots, T_{2 n}$,

$$
T_{j}=(-1)^{j} \operatorname{det}\left(\begin{array}{ccccc}
A_{1,0}^{\dagger} & \cdots & \widehat{A}_{1, j}^{\dagger} & \cdots & A_{1,2 n}^{\dagger} \\
\vdots & & \vdots & & \vdots \\
A_{2 n, 0}^{\dagger} & \cdots & \widehat{A}_{2 n, j}^{\dagger} & \cdots & A_{2 n, 2 n}^{\dagger}
\end{array}\right), \quad j=0,1, \ldots, 2 n
$$

where $\widehat{A}_{i, j}^{\dagger}$ means the omission of the $(j+1)$-th column of $2 n \times(2 n+1)$ array for $A_{i, j}^{\dagger}$.

Since all coefficients of $A_{i, 0}, i=1,2, \ldots, 2 n$ in (2.7) are equal to 1 , we can use the approach similar to [21] and Theorem 2.1 and obtain the following theorem.

Theorem 2.3. If the given eigenvalues $\lambda_{1}, \lambda_{2}, \ldots, \lambda_{2 n}$ are distinct, then the solutions of PQIEP coincide with the simultaneous eigenvalues of the following joint eigenvalue problem:

$$
\begin{aligned}
& \left(T_{1}-c_{1} T_{0}\right) x=0, \\
& \left(T_{2}-c_{2} T_{0}\right) x=0, \\
& \cdots \cdots \cdots \cdots \cdots \cdots \cdots \\
& \left(T_{2 n}-c_{2 n} T_{0}\right) x=0 .
\end{aligned}
$$

Using Theorems 2.1 and 2.3, we derive a sufficient condition for the solvability of PQIEP.

Theorem 2.4. If the given eigenvalues $\lambda_{1}, \lambda_{2}, \ldots, \lambda_{2 n}$ are distinct and $T_{0}$ is invertible, then PQIEP has at least one solution.

Proof. The proof is similar to that of [11, Theorem 2.4] and it is omitted here. 


\section{Smooth $Q R$-Decomposition-Based Newton Method for PQIEP}

In order to construct a numerical method for finding a solution $c^{*}$ of PQIEP, we recall the smooth $Q R$-decomposition with column pivoting for a matrix-valued function depending on several parameters - cf. $[13,25]$ for more details.

Let $B(c)=\left(b_{k l}(c)\right) \in \mathbb{C}^{n \times n}$ be a twice continuously differentiable matrix-valued function defined on an open connected domain $\mathbf{D} \subseteq \mathbb{C}^{2 n}$. Here, the twice differentiability of $B(c)$ with respect to $c=\left(c_{1}, c_{2}, \ldots, c_{2 n}\right)^{T}$ means that for any $c^{(0)}=\left(c_{1}^{(0)}, c_{2}^{(0)}, \ldots, c_{2 n}^{(0)}\right)^{T} \in \mathbf{D}$ the partial derivatives $\partial b_{k l}(c) / \partial c_{i}, k, l=1, \ldots, n, i=1, \ldots, 2 n$ exist and

$$
B(c)=B\left(c^{(0)}\right)+\sum_{i=1}^{2 n} \frac{\partial B\left(c^{(0)}\right)}{\partial c_{i}}\left(c_{i}-c_{i}^{(0)}\right)+\mathscr{O}\left(\left\|c-c^{(0)}\right\|_{2}^{2}\right)
$$

where

$$
\frac{\partial B\left(c^{(0)}\right)}{\partial c_{i}}=\left(\left.\frac{\partial b_{k l}(c)}{\partial c_{i}}\right|_{c=c^{(0)}}\right) \in \mathbb{C}^{n \times n} .
$$

The following results is obtained from [13, Theorem 3.2], concerns the existence of a locally smooth $Q R$-decomposition of $B(c)$.

Theorem 3.1. Let $B(c) \in \mathbb{C}^{n \times n}$ be twice continuously differentiable at $c^{(0)} \in \mathbf{D} \subseteq \mathbb{C}^{2 n}$. Assume that $\operatorname{rank}\left(\mathrm{B}\left(\mathrm{c}^{(0)}\right)\right) \geq \mathrm{n}-1, \Pi \in \mathbb{C}^{n \times n}$ is a permutation matrix such that the first $n-1$ columns of $B\left(c^{(0)}\right) \Pi$ are linearly independent, and $B\left(c^{(0)}\right) \Pi$ has the $Q R$-decomposition

$$
B\left(c^{(0)}\right) \Pi=Q_{0} R_{0},
$$

where $Q_{0} \in \mathscr{U}_{n}$

$$
R_{0}=\left(\begin{array}{cc}
R_{11} & R_{12} \\
0 & r_{n n}
\end{array}\right)
$$

and $R_{11} \in \mathscr{R}_{n-1}$ is a nonsingular matrix. Then there exists a neighborhood $\mathscr{N}\left(c^{(0)}\right) \subset \mathbf{D}$ of $c^{(0)}$ such that for all $c \in \mathscr{N}\left(c^{(0)}\right)$ the matrix-valued function $B(c) \Pi$ has the QR-decomposition

$$
B(c) \Pi=Q(c) R(c),
$$

where $Q(c) \in \mathscr{U}_{n}$

$$
R(c)=\left(\begin{array}{cc}
R_{11}(c) & R_{12}(c) \\
0 & r_{n n}(c)
\end{array}\right)
$$

and $R_{11}(c) \in \mathscr{R}_{n-1}$. Moreover, this $Q R$-decomposition has the following properties:

(1) $Q\left(c^{(0)}\right)=Q_{0}$ and $R\left(c^{(0)}\right)=R_{0}$.

(2) All elements of $Q(c)$ and $R(c)$ are continuous in $\mathscr{N}\left(c^{(0)}\right)$.

(3) The diagonal elements of $R(c)$ are continuously differentiable at $c^{(0)}$, and

$$
r_{n n}(c)=r_{n n}+\left.e_{n}^{T} Q_{0}^{H} \sum_{i=1}^{2 n} \frac{\partial B(c)}{\partial c_{i}}\right|_{c=c^{(0)}} \Pi\left(e_{n}-I^{(n-1)} R_{11}^{-1} R_{12}\right)\left(c_{i}-c_{i}^{(0)}\right)+\mathscr{O}\left(\left\|c-c^{(0)}\right\|_{2}^{2}\right) .
$$


Since $C(c)$ and $K(c)$ are the affine families (1.4) and $\lambda_{1}, \lambda_{2}, \ldots, \lambda_{2 n}$ are distinct, the functions $Q_{c}\left(\lambda_{i}\right)=\lambda_{i}^{2} M+\lambda_{i} C(c)+K(c), i=1,2, \ldots, 2 n$ are twice continuously differentiable in $\mathbb{C}^{2 n}$, and $\operatorname{rank}\left[\mathrm{Q}_{\mathrm{c}^{*}}\left(\lambda_{\mathrm{i}}\right)\right]=\mathrm{n}-1, i=1,2, \ldots, 2 \mathrm{n}$. It follows from Theorem 3.1 that there are a neighborhood $\mathscr{N}\left(c^{*}\right) \subset \mathbb{C}^{2 n}$ of $c^{*}$ and permutation matrices $\Pi_{i}\left(c^{*}\right) \in \mathbb{C}^{n \times n}$, $i=1,2, \ldots, 2 n$ such that

$$
Q_{c^{*}}\left(\lambda_{i}\right) \Pi_{i}\left(c^{*}\right)=Q_{i}\left(c^{*}\right) R_{i}\left(c^{*}\right),
$$

where $Q_{i}\left(c^{*}\right) \in \mathscr{U}_{n}$

$$
R_{i}\left(c^{*}\right)=\left(\begin{array}{cc}
R_{11}^{(i)}\left(c^{*}\right) & R_{12}^{(i)}\left(c^{*}\right) \\
0 & 0
\end{array}\right)
$$

and $R_{11}^{(i)}\left(c^{*}\right) \in \mathscr{R}_{n-1}$ is a nonsingular matrix.

For any $c \in \mathscr{N}\left(c^{*}\right)$, we compute the $Q R$-decompositions of $\lambda_{i}^{2} M+\lambda_{i} C(c)+K(c), i=$ $1,2, \ldots, 2 n$ with column pivoting as follows:

$$
\left[\lambda_{i}^{2} M+\lambda_{i} C(c)+K(c)\right] \Pi_{i}(c)=Q_{i}(c) R_{i}(c), \quad i=1,2, \ldots, 2 n,
$$

where $\Pi_{i}(c)$ is a permutation matrix, $Q_{i}(c) \in \mathscr{U}_{n}$

$$
R_{i}(c)=\left(\begin{array}{cc}
R_{11}^{(i)}(c) & R_{12}^{(i)}(c) \\
0 & r_{n n}^{(i)}(c)
\end{array}\right)
$$

and $R_{11}^{(i)}(c) \in \mathscr{R}_{n-1}$. Assume that $\mathscr{N}\left(c^{*}\right)$ is sufficiently small, so that for each $i$ the permutation matrices $\Pi_{i}(c), i=1,2, \ldots, 2 n$ are constant and the column pivoting is performed. The matrices $R_{11}^{(i)}(c), i=1,2, \ldots, 2 n$ are nonsingular since the prescribed eigenvalues $\lambda_{1}, \lambda_{2}, \ldots, \lambda_{2 n}$ are simple. Then the quadratic eigenvalue problem (1.3) has the eigenvalues $\lambda_{1}, \lambda_{2}, \ldots, \lambda_{2 n}$ if and only if $c \in \mathbb{C}^{2 n}$ satisfies the equations

$$
r_{n n}^{(i)}(c)=0, \quad i=1,2, \ldots, 2 n \text {. }
$$

Let

$$
f(c)=\left(\begin{array}{c}
r_{n n}^{(1)}(c) \\
r_{n n}^{(2)}(c) \\
\vdots \\
r_{n n}^{(2 n)}(c)
\end{array}\right)
$$

Then $c^{*} \in \mathbb{C}^{2 n}$ is a solution of PQIEP if and only if $c^{*}$ is a solution of the system of nonlinear equations

$$
f(c)=0 .
$$

Let $c^{(k)} \in \mathscr{N}\left(c^{*}\right)$ be the current approximation to the solution $c^{*}$ of the nonlinear system (3.2). It follows from Theorem 3.1 that the functions $r_{n n}^{(i)}(c), i=1,2, \ldots, 2 n$ are continuously differentiable at $c^{(k)}$ and they can be represented in the form

$$
r_{n n}^{(i)}(c)=r_{n n}^{(i)}\left(c^{(k)}\right)+\sum_{j=1}^{2 n} \frac{\partial r_{n n}^{(i)}\left(c^{(k)}\right)}{\partial c_{j}}\left(c_{j}-c_{j}^{(k)}\right)+\mathscr{O}\left(\left\|c_{j}-c_{j}^{(k)}\right\|_{2}^{2}\right), \quad j=1,2, \ldots, 2 n,
$$


where

$$
\begin{aligned}
\frac{\partial r_{n n}^{(i)}\left(c^{(k)}\right)}{\partial c_{j}}= & e_{n}^{T} Q_{i}\left(c^{(k)}\right)^{H}\left(\lambda_{i} C_{j}+K_{j}\right) \Pi_{i}\left(c^{(k)}\right) \\
& \times\left[e_{n}-I^{(n-1)}\left(R_{11}^{(i)}\left(c^{(k)}\right)\right)^{-1} R_{12}^{(i)}\left(c^{(k)}\right)\right] .
\end{aligned}
$$

Applying the Newton method [27] to the nonlinear system (3.2) and using (3.3), we obtain the following Newton iteration:

$$
J_{f}\left(c^{(k)}\right)\left(c^{(k+1)}-c^{(k)}\right)=-f\left(c^{(k)}\right),
$$

where

$$
J_{f}(c)=\left(\frac{\partial r_{n n}^{(i)}(c)}{\partial c_{j}}\right)
$$

is the Jacobian matrix of the nonlinear function $f(c)$. Thus a new method for solving PQIEP may be summarised as follows.

Now we consider the computational cost of Algorithm 3.1. Step 1 needs $8 n^{3}$ flops. The $Q R$-decomposition of $\lambda_{i}^{2} M+\lambda_{i} C\left(c^{(k)}\right)+K\left(c^{(k)}\right)$ by using Householder transformations

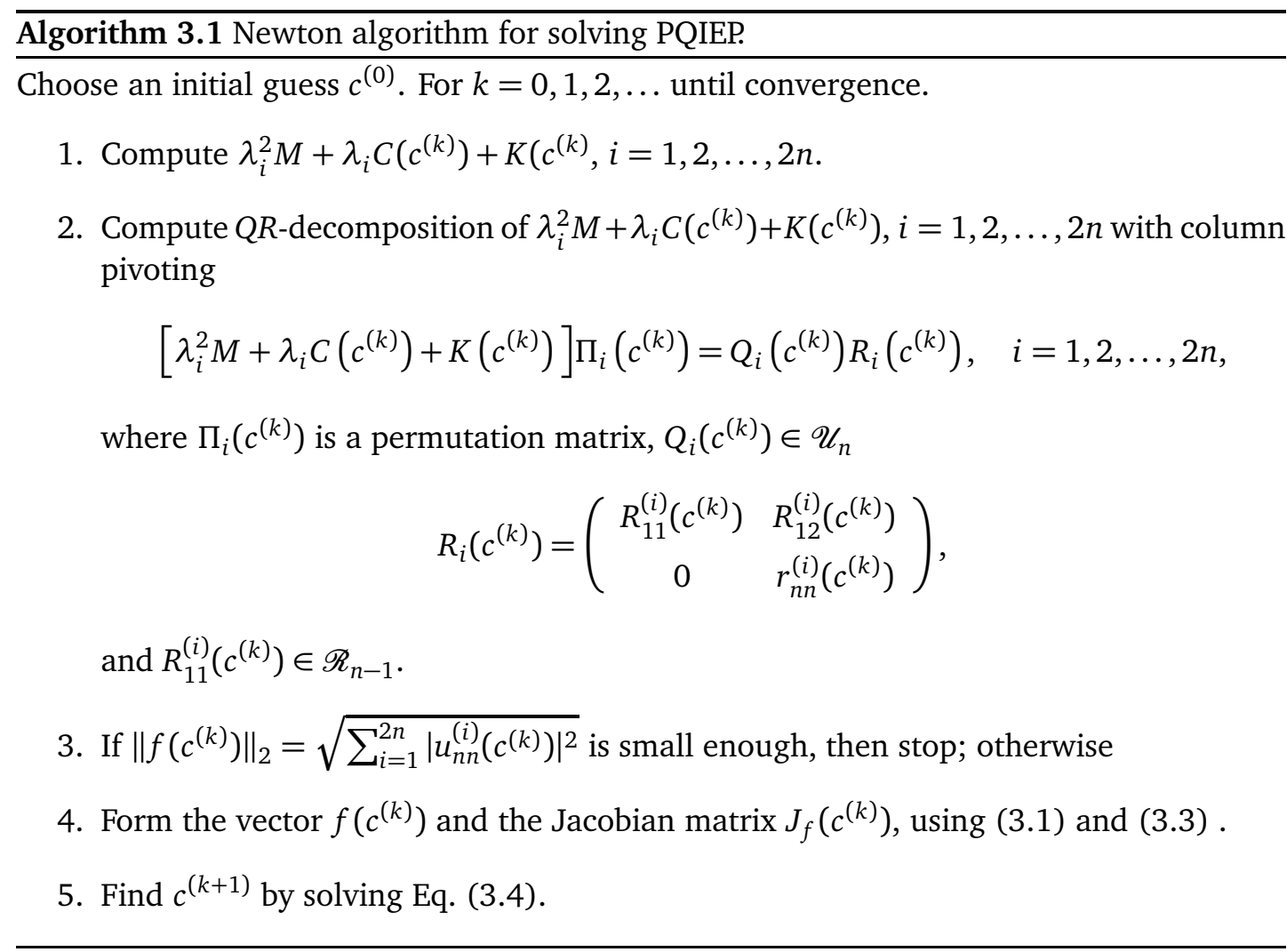


with column pivoting requires (4/3) $n^{3}$ flops - cf. [19], so Step 2 needs $(8 / 3) n^{4}$ flops. The computational cost of Step 3 is negligible. Step 4 requires approximately $14 n^{4}$ flops. Step 5 needs $(16 / 3) n^{3}$ flops if the linear system in (3.4) is solved by the Gaussian elimination with partial pivoting. Therefore Algorithm 3.1 requires approximately $(2 / 3)\left(25 n^{4}+20 n^{3}\right)$ flops per iteration, where forming the Jacobian matrix $J_{f}\left(c^{(k)}\right)$ accounts for most of the computational cost.

In general, the $Q R$-decompositions of $\lambda_{i}^{2} M+\lambda_{i} C\left(c^{(k)}\right)+K\left(c^{(k)}\right), i=1,2, \ldots, 2 n$ with column pivoting are not unique. Similar to [9, Theorem 3.1], it can be shown that the iterates $c^{(k)}$ generated by Algorithm 3.1 are always same corresponding to different $Q R$ decompositions with column pivoting. The convergence of Algorithm 3.1 can be described by the following theorem.

Theorem 3.2. Suppose that PQIEP has a solution $c^{*}$ and $\Pi_{i}\left(c^{(k)}\right)=\Pi_{i}\left(c^{*}\right)(i=1,2, \ldots, 2 n)$ used in Algorithm 3.1 do not depend on $k$ for sufficiently small $\left\|c^{*}-c^{(k)}\right\|_{2}$. If the Jacobian matrix $J_{f}\left(c^{*}\right)$ corresponding to the $Q R$-decompositions of $\left(\lambda_{i}^{2} M+\lambda_{i} C\left(c^{*}\right)+K\left(c^{*}\right)\right) \Pi_{i}\left(c^{*}\right), i=$ $1,2, \ldots, 2 n$ is nonsingular, then Algorithm 3.1 is locally quadratically convergent.

Proof. The proof is similar to that of [9, Theorem 3.2]. Hence, it is omitted.

\section{Numerical Examples}

In this section, we present the results of numerical experiments, which show the efficiency of the proposed method in finding approximate solutions of PQIEPs. All experiments are performed in MATLAB R2019b with double precision arithmetic on an Intel Core 2.9 GHz PC with 4GB memory under Windows 10 system. All iterations start with initial guesses close to the exact solution $c^{*}$ of PQIEP, so that only a few iterations are required for convergence. In the tables, CPU denotes the computing time (in seconds) spent on establishing the corresponding approximate solution.

Example 4.1. Consider the dynamic behavior control of a damped mass-spring system. The original damped mass-spring system is shown in Fig. 1. The mass, damping and stiffness matrices of the system are

$$
M=\left(\begin{array}{lll}
1 & 0 & 0 \\
0 & 2 & 0 \\
0 & 0 & 3
\end{array}\right), \quad C_{0}=\left(\begin{array}{ccc}
3 & -2 & 0 \\
-2 & 3 & -1 \\
0 & -1 & 1
\end{array}\right), \quad K_{0}=\left(\begin{array}{ccc}
8 & -4 & 0 \\
-4 & 11 & -7 \\
0 & -7 & 7
\end{array}\right)
$$

We want to modify the system so that it will have the desired dynamic behavior. The first and second springs are perturbed by $k_{1}$ and $k_{2}$, respectively. The first and second dampers are perturbed by $d_{1}$ and $d_{2}$, respectively. The first and third masses are linked by the spring $k_{3}$ and the damper $d_{3}$. The modified damped mass-spring system is shown in Fig. 2. We choose the parameters $k_{1}, k_{2}, k_{3}$ and $d_{1}, d_{2}, d_{3}$ so that the modified system has the eigenvalues

$$
\begin{array}{lll}
\lambda_{1}=-3+\mathrm{i}, & \lambda_{2}=-3-\mathrm{i}, & \lambda_{3}=-3+2 \mathrm{i}, \\
\lambda_{4}=-3-2 \mathrm{i}, & \lambda_{5}=-3+3 \mathrm{i}, & \lambda_{6}=-3-3 \mathrm{i},
\end{array}
$$




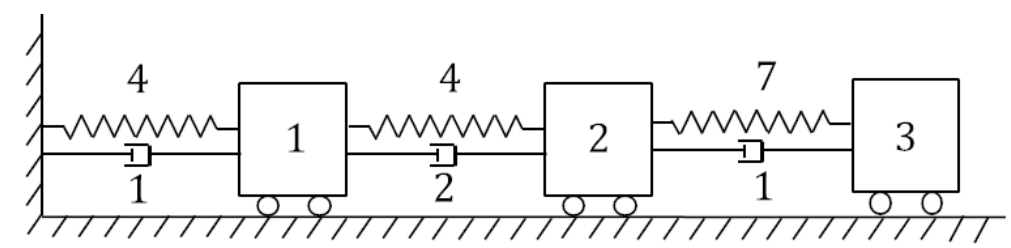

Figure 1: Original damped mass-spring system.

where $i$ is the imaginary unit. The mass, damping and stiffness matrices of the modifying system may be expressed as

$$
M=\left(\begin{array}{lll}
1 & 0 & 0 \\
0 & 2 & 0 \\
0 & 0 & 3
\end{array}\right), \quad C(c)=C_{0}+\sum_{i=1}^{6} c_{i} C_{i}, \quad K(c)=K_{0}+\sum_{i=1}^{6} c_{i} K_{i}
$$

where $c_{i}=d_{i}, c_{3+i}=k_{i}, i=1,2,3$, and

$$
C_{1}=K_{4}=\left(\begin{array}{lll}
1 & 0 & 0 \\
0 & 0 & 0 \\
0 & 0 & 0
\end{array}\right), \quad C_{2}=K_{5}=\left(\begin{array}{ccc}
1 & -1 & 0 \\
-1 & 1 & 0 \\
0 & 0 & 0
\end{array}\right), \quad C_{3}=K_{6}=\left(\begin{array}{ccc}
1 & 0 & -1 \\
0 & 0 & 0 \\
-1 & 0 & 1
\end{array}\right),
$$

$C_{4}=C_{5}=C_{6}=K_{1}=K_{2}=K_{3}=O$ are the $3 \times 3$ zero matrices.

Our goal is to determine a vector $c^{*}=\left(d_{1}, d_{2}, d_{3}, k_{1}, k_{2}, k_{3}\right)^{T}$ such that the modified damped mass-spring system has the prescribed eigenvalues. With the initial value

$$
I: c^{(0)}=(10,0,0,10,10,50)^{T}
$$

Algorithm 3.1 and the algorithm in [16] both converge to the solution

$$
c^{*}=(14.0461,-0.7005,0.1286,8.1518,4.6088,70.1606)^{T} .
$$

With another starting value

$$
I I: c^{(0)}=(10,0,0,10,50,10)^{T},
$$

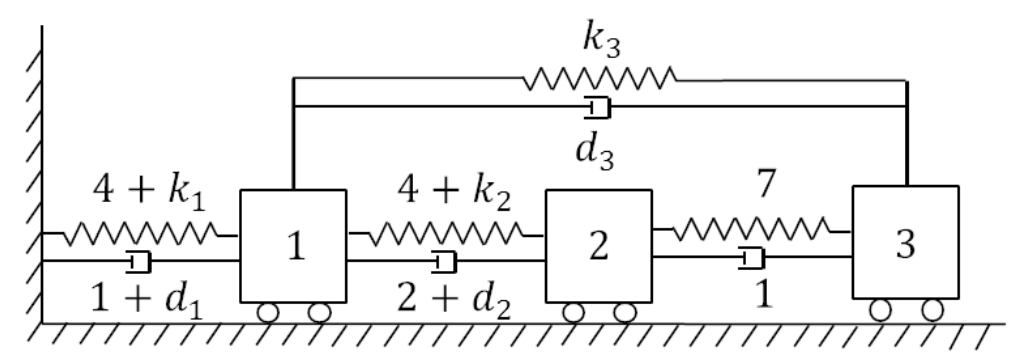

Figure 2: Modified damped mass-spring system. 
Table 1: Numerical results for Example 4.1.

\begin{tabular}{||c|c|c|c|c|c||}
\hline \multicolumn{2}{||c|}{} & \multicolumn{2}{|c|}{ Algorithm 3.1} & \multicolumn{2}{c||}{ Algorithm in [16] } \\
\hline$c^{(0)}$ & $k$ & $\left\|c^{(k+1)}-c^{(k)}\right\|_{2}$ & $\left\|f\left(c^{(k)}\right)\right\|_{2}$ & $\left\|c^{(k+1)}-c^{(k)}\right\|_{2}$ & $\left\|f\left(c^{(k)}\right)\right\|_{2}$ \\
\hline \multirow{5}{*}{ I } & 0 & $1.12 e+01$ & $3.35 e+10$ & $2.62 e+01$ & $9.43 e+04$ \\
\cline { 2 - 6 } & 1 & $1.30 e+01$ & $4.88 e-01$ & $5.63 e+00$ & $3.28 e+04$ \\
\cline { 2 - 6 } & 2 & $1.24 e+00$ & $1.11 e+00$ & $3.43 e-01$ & $1.72 e+03$ \\
\cline { 2 - 6 } & 3 & $3.55 e-02$ & $3.50 e-02$ & $5.70 e-03$ & $2.09 e+01$ \\
\cline { 2 - 6 } & 4 & $1.42 e-04$ & $2.03 e-05$ & $7.80 e-07$ & $1.65 e-03$ \\
\cline { 2 - 6 } & 5 & $4.60 e-10$ & $6.61 e-11$ & $1.42 e-12$ & $1.40 e-10$ \\
\hline CPU & & \multicolumn{2}{|c|}{0.0088} & \multicolumn{2}{c||}{0.0721} \\
\hline \multirow{4}{*}{ II } & 0 & $6.22 e+00$ & $2.28 e+01$ & $8.72 e+00$ & $8.73 e+04$ \\
\cline { 2 - 6 } & 1 & $1.11 e+00$ & $5.79 e-01$ & $2.20 e+00$ & $1.71 e+04$ \\
\cline { 2 - 6 } & 2 & $1.04 e-01$ & $1.82 e-02$ & $1.65 e-01$ & $9.02 e+02$ \\
\cline { 2 - 6 } & 3 & $2.91 e-05$ & $5.61 e-05$ & $7.02 e-04$ & $2.17 e+00$ \\
\cline { 2 - 6 } & 4 & $1.00 e-10$ & $4.17 e-11$ & $9.33 e-09$ & $1.03 e-05$ \\
\hline CPU & & \multicolumn{2}{|c|}{0.0076} & \multicolumn{2}{c||}{0.0079} \\
\hline
\end{tabular}

Algorithm 3.1 and the algorithm in [16] both converge to the solution

$$
c^{*}=(3.1640,-0.3249,0.3675,6.9453,53.2345,13.7325)^{T} .
$$

Both Algorithm 3.1 and the algorithm in [16] stop if

$$
\left\|c^{(k+1)}-c^{(k)}\right\|_{2} \leq 10^{-8} .
$$

Table 1 shows that Algorithm 3.1 and the algorithm in [16] have locally quadratic convergence rate, but in terms of the computing time, Algorithm 3.1 is more effective than the one in [16].

Example 4.2. Let $n=5, M=I$,

$$
\begin{aligned}
& C_{0}=K_{0}=\left(\begin{array}{rrrrr}
10 & -10 & 0 & 0 & 0 \\
-10 & 18 & -8 & 0 & 0 \\
0 & -8 & 12 & -4 & 0 \\
0 & 0 & -8 & 12 & -4 \\
0 & 0 & 0 & -8 & 11
\end{array}\right), \\
& C_{i}=K_{n+i}=\left(e_{i}-e_{i+1}\right)\left(e_{i}-e_{i+1}\right)^{T}, \quad i=1,2, \ldots, n-1, \\
& C_{n}=K_{2 n}=e_{1} e_{1}^{T}+e_{n} e_{n}^{T}, \\
& C_{n+i}=K_{i}=O, \quad i=1,2, \ldots, n .
\end{aligned}
$$

The quadratic pencil $Q_{c}(\lambda)=\lambda^{2} M+\lambda C(c)+K(c)$ at $c^{*}=(-1,1,-1,1,-1,1,-1,1,-1,1)^{T}$ has real eigenvalues $-26.0740,-18.4743,-8.9137,-2.4879,-1.1160,-0.3637$ and complex eigenvalues $-1.6923 \pm 2.4350 \mathrm{i},-0.0929 \pm 0.7285 \mathrm{i}$. 
In this example, we compare Algorithm 3.1 with the algorithms in [16] and [17]. In order to keep the number of real and complex eigenvalues in the iterative process of the algorithm in [17] the same as the number of real and complex eigenvalues prescribed, we choose an initial guess $c^{(0)}$ close to the exact solution $c^{*}$ of PQIEP. All algorithms stop if

$$
\left\|c^{(k)}-c^{*}\right\|_{2} \leq 10^{-8} \text {. }
$$

With the starting value

$$
c^{(0)}=(-0.9724,1.0680,-0.9345,1.0163,-0.9881,1.0498,-0.9040,1.0340,-0.9415,1.0224)^{T}
$$

Algorithm 3.1 and the algorithms in [16] and [17] converge to the exact solution $c^{*}$ of PQIEP. The numerical results for Algorithm 3.1 and the algorithms in [16] and [17] are reported in Table 2 . We observe that all three algorithms are all effective and locally quadratically convergent. Both Algorithm 3.1 and the one in [17] take about the same computing time, which is lower than that of the algorithm in [16].

\begin{tabular}{|c|c|c|c|c|c|c|}
\hline & \multicolumn{2}{|c|}{ Algorithm 3.1} & \multicolumn{2}{|c|}{ Algorithm in [17] } & \multicolumn{2}{|c|}{ Algorithm in [16] } \\
\hline$k$ & $\left\|c^{(k)}-c^{*}\right\|_{2}$ & $\left\|f\left(c^{(k)}\right)\right\|_{2}$ & $\left\|c^{(k)}-c^{*}\right\|_{2}$ & $\left\|f\left(c^{(k)}\right)\right\|_{2}$ & $\left\|c^{(k)}-c^{*}\right\|_{2}$ & $\left\|f\left(c^{(k)}\right)\right\|_{2}$ \\
\hline 0 & $1.64 e-01$ & $5.94 e+00$ & $1.64 e-01$ & $2.06 e-01$ & $1.64 e-01$ & $1.52 e+11$ \\
\hline 1 & $2.44 e-03$ & $1.54 e-02$ & $1.73 e-03$ & $3.76 e-04$ & $1.79 e-02$ & $2.51 e+09$ \\
\hline 2 & $2.50 e-05$ & $7.06 e-06$ & $1.94 e-06$ & $1.76 e-07$ & $2.21 e-03$ & $9.93 e+05$ \\
\hline 3 & $3.02 e-09$ & $8.42 e-10$ & $2.01 e-11$ & $2.72 e-13$ & $2.28 e-05$ & $1.95 e+03$ \\
\hline 4 & - & - & - & - & $2.47 e-09$ & $2.05 e-01$ \\
\hline CPU & \multicolumn{2}{|c|}{0.0432} & \multicolumn{2}{|c|}{0.0455} & \multicolumn{2}{|c|}{0.0634} \\
\hline
\end{tabular}

Table 2: Numerical results for Example 4.2.

Example 4.3. To construct test examples of PQIEPs, the elements of nonsymmetric matrices $M, C=\left(c_{s t}\right) \in \mathbb{R}^{n \times n}$ are generated from a uniform distribution in the interval $(-2,2)$, the off-diagonal elements of nonsymmetric matrix $K=\left(k_{s t}\right) \in \mathbb{R}^{n \times n}$ from a uniform distribution in the interval $(-1,1)$, and the diagonal elements of the matrix $K$ from a uniform distribution in the interval $(0,200)$. Computing the eigenvalues $\lambda_{1}, \lambda_{2}, \ldots, \lambda_{2 n}$ of the quadratic pencil $Q(\lambda)=\lambda^{2} M+\lambda C+K$ and setting

$$
\begin{aligned}
& C_{0}=0, \quad C_{1}=\sum_{s=1}^{n} c_{s s} e_{s} e_{s}^{T}, \quad C_{n+i}=O, \quad i=1, \ldots, n, \\
& C_{i}=\sum_{s=1}^{n-i+1}\left(c_{s, s+i-1} e_{s} e_{s+i-1}^{T}+c_{s+i-1, s} e_{s+i-1} e_{s}^{T}\right), \quad i=2, \ldots, n, \\
& K_{0}=K_{i}=O, \quad i=1, \ldots, n, \quad K_{n+1}=\sum_{s=1}^{n} k_{s s} e_{s} e_{s}^{T},
\end{aligned}
$$




$$
K_{n+i}=\sum_{s=1}^{n-i+1}\left(k_{s, s+i-1} e_{s} e_{s+i-1}^{T}+k_{s+i-1, s} e_{s+i-1} e_{s}^{T}\right), \quad i=2, \ldots, n,
$$

we then recompute the exact solution $c^{*}=(1,1, \ldots, 1) \in \mathbb{R}^{2 n}$ of PQIEP by making use of the eigenvalues obtained.

The initial point $c^{(0)}$ is obtained by $c^{*}$ plus $1 \%$ noise uniformly distributed in the interval $[0,1]$. Both Algorithm 3.1 and the algorithm in [16] converge to $c^{*}$ locally and quadratically. All algorithms are terminated if

$$
\left\|c^{(k)}-c^{*}\right\|_{2} \leq 10^{-5}
$$

The numerical results presented in Table 3 show that Algorithm 3.1 is more effective than the one in [16] in terms of computing time and numerical stability. We failed to compare Algorithm 3.1 with the algorithm in [17] since it is difficult to choose the initial point so that the number of real and complex eigenvalues in the iterative process of the algorithm in [17] remains the same as the number of real and complex eigenvalues prescribed.

Table 3: Numerical results for Example 4.3.

\begin{tabular}{||c|c|c|c|c|c||}
\hline \multicolumn{2}{||c|}{} & \multicolumn{3}{|c|}{ Algorithm 3.1 } & \multicolumn{2}{c||}{ Algorithm in [16] } \\
\hline$n$ & $k$ & $\left\|c^{(k)}-c^{*}\right\|_{2}$ & $\left\|f\left(c^{(k)}\right)\right\|_{2}$ & $\left\|c^{(k)}-c^{*}\right\|_{2}$ & $\left\|f\left(c^{(k)}\right)\right\|_{2}$ \\
\hline \multirow{5}{*}{50} & 0 & $6.10 e-02$ & $9.20 e-01$ & $6.10 e-02$ & $1.38 e+144$ \\
\cline { 2 - 6 } & 1 & $1.91 e-02$ & $2.92 e-03$ & $1.24 e-04$ & $5.46 e+141$ \\
\cline { 2 - 6 } & 2 & $5.23 e-05$ & $3.77 e-06$ & $3.23 e-07$ & $4.59 e+137$ \\
\cline { 2 - 6 } & 3 & $6.11 e-10$ & $1.89 e-11$ & $2.22 e-10$ & $5.66 e+132$ \\
\hline CPU & & \multicolumn{2}{|c|}{3.6459} & \multicolumn{2}{|c||}{23.8648} \\
\hline \multirow{5}{*}{100} & 0 & $8.15 e-02$ & $1.73 e+01$ & $8.15 e-02$ & overflow \\
\cline { 2 - 6 } & 1 & $4.91 e-02$ & $7.17 e-03$ & $9.74 e-02$ & overflow \\
\cline { 2 - 6 } & 2 & $2.35 e-04$ & $2.10 e-05$ & $2.05 e-04$ & overflow \\
\cline { 2 - 6 } & 3 & $7.17 e-09$ & $6.92 e-10$ & $5.33 e-07$ & overflow \\
\hline CPU & & \multicolumn{2}{|c|}{47.0159} & \multicolumn{2}{|c||}{157.4417} \\
\hline \multirow{4}{*}{150} & 0 & $1.03 e-01$ & $2.61 e+00$ & $1.03 e-01$ & overflow \\
\cline { 2 - 6 } & 1 & $1.03 e-01$ & $1.22 e-02$ & $8.16 e-01$ & overflow \\
\cline { 2 - 6 } & 2 & $9.51 e-04$ & $9.70 e-05$ & $6.57 e-04$ & overflow \\
\cline { 2 - 6 } & 3 & $1.54 e-07$ & $1.65 e-08$ & $1.21 e-07$ & overflow \\
\hline CPU & & \multicolumn{2}{|c|}{203.8927} & \multicolumn{2}{|c||}{810.3174} \\
\hline \multirow{3}{*}{200} & 0 & $1.11 e-01$ & $4.90 e+00$ & $1.11 e-01$ & overflow \\
\cline { 2 - 6 } & 1 & $1.65 e-01$ & $1.79 e-02$ & $1.58 e-01$ & overflow \\
\cline { 2 - 6 } & 2 & $5.10 e-03$ & $3.59 e-04$ & $4.40 e-03$ & overflow \\
\cline { 2 - 6 } & 3 & $5.36 e-06$ & $1.87 e-07$ & $3.76 e-06$ & overflow \\
\hline CPU & & \multicolumn{3}{|c|}{632.4458} & 2742.1762 \\
\hline
\end{tabular}




\section{Conclusion}

We showed that if the given eigenvalues are distinct, then PQIEP is equivalent to a multiparameter eigenvalue problem and presented a sufficient condition for the PQIEP solvability. In order to find approximate solution of the PQIEP, we employed the Newton method based on the smooth $Q R$-decomposition with column pivoting and proved its locally quadratic convergence. The computational cost of this algorithm is dominated by forming the Jacobian matrix at each iteration. This approach is also applicable to PQIEP with analytical matrix-valued functions $M(c), C(c)$ and $K(c)$ with $c \in \mathbb{R}^{2 n}$ or $c \in \mathbb{C}^{2 n}$, and its efficiency can be improved by using the quasi-Newton method.

\section{Acknowledgments}

The authors are very much indebted to anonymous referees and to the Editors of EAJAM, Tao Zhou and Victor Didenko, for their useful comments and suggestions.

This work is supported by the National Natural Science Foundation of China under Grant No. 11571171.

\section{References}

[1] A.L. Andrew, K.-W. E. Chu and P. Lancaster, Derivatives of eigenvalues and eigenvectors of matrix functions, SIAM J. Matrix Anal. Appl. 14, 903-926 (1993).

[2] F.V. Atkinson, Multiparameter Eigenvalue Problems. Volume I: Matrices and Compact Operators, Academic Press (1972).

[3] F.W. Biegler-König, A Newton iteration process for inverse eigenvalue problems, Numer. Math. 37, 349-354 (1981).

[4] Z. Bohte, Numerical solution of the inverse algebraic eigenvalue problem, Computer J. 10, 385388 (1968).

[5] R.H. Chan, S.-F. Xu and H.-M. Zhou, On the convergence rate of a quasi-Newton method for inverse eigenvalue problems, SIAM J. Numer. Anal. 36, 436-441 (1999).

[6] M.T. Chu and G.H. Golub, Inverse Eigenvalue Problems: Theory, Algorithms, and Applications, Oxford University Press (2005).

[7] N. Cottin, Dynamic model updating - a multiparameter eigenvalue problem, Mech. Syst. and Signal Pr. 15, 649-665 (2001).

[8] N. Cottin and J. Reetz, Accuracy of multiparameter eigenvalues used for dynamic model updating with measured natural frequencies only, Mech. Syst. and Signal Pr. 20, 65-77 (2006).

[9] H. Dai, An algorithm for symmetric generalized inverse eigenvalue problems, Linear Algebra Appl. 296, 79-98 (1999).

[10] H. Dai, The Theory of Matrices (in Chinese), Science Press (2001).

[11] H. Dai, Z.-Z. Bai and Y. Wei, On the solvability condition and numerical algorithm for the parameterized generalized inverse eigenvalue problem, SIAM J. Matrix Anal. Appl. 36, 707-726 (2015).

[12] H. Dai and P. Lancaster, Newton's method for a generalized inverse eigenvalue problem, Numer. Linear Algebra Appl. 4, 1-21 (1997).

[13] H. Dai and P. Lancaster, Numerical methods for finding multiple eigenvalues of matrices depending on parameters, Numer. Math. 76, 189-208 (1997). 
[14] B.N. Datta and V. Sokolov, A solution of the affine quadratic inverse eigenvalue problem, Linear Algebra Appl. 434, 1745-1760 (2011).

[15] A.C. Downing and A.S. Householder, Some inverse characteristic value problems, J. Assoc. Comput. Mach. 3, 203-207 (1958).

[16] S. Elhay and Y. M. Ram, An affine inverse eigenvalue problem, Inverse Problems 18, 455-466 (2002).

[17] S. Elhay and Y.M. Ram, Quadratic pencil pole assignment by affine summs, ANZIAM J. 45, 592-603 (2004).

[18] S. Friedland, J. Nocedal and M.L. Overton, The formulation and analysis of numerical methods for inverse eigenvalue problems, SIAM J. Numer. Anal. 24, 634-667 (1987).

[19] G.H. Golub and C.F. Van Loan, Matrix Computations, Johns Hopkins University Press (1996).

[20] K.P. Hadeler, Newton-Verfahren für inverse Eigenwertaufgaben, Numer. Math. 12, 35-39 (1968).

[21] X. Ji, On matrix inverse eigenvalue problems, Inverse Problems 14, 275-285 (1998).

[22] K.T. Joseph, Inverse eigenvalue problem in structural design, AIAA J. 30, 2890-2896 (1992).

[23] H.W. Kuhn, The Hungarian method for the assignment problem, Naval Res. Logist. Quart. 2, 83-97 (1955).

[24] P. Lancaster, Algorithms for lambda-matrices, Numer. Math. 6, 388-394 (1964).

[25] R.-C. Li, QR-decomposition and nonlinear eigenvalue problems (in Chinese), Math. Numer. Sinica 11, 374-385 (1989).

[26] R.-C. Li, Algorithms for inverse eigenvalue problems, J. Comput. Math. 10, 97-111 (1992).

[27] M. Ortega and W.C. Rheinboldt, Iterative Solution of Nonlinear Equations in Several Variables, Academic Press (1970).

[28] W. Shen, C. Li and J.-C. Yao, Convergence analysis of Newton-like methods for inverse eigenvalue problems with multiple eigenvalues, SIAM J. Numer. Anal. 54, 2938-2950 (2016).

[29] Q. Song and H. Dai, Numerical methods for solving generalized inverse eigenvalue problem (in Chinese), Journal of Nanjing University of Aeronautics and Astronautics 31, 679-685 (1999).

[30] D. Sun and J. Sun, Strong semismoothness of symmetric matrices and its application to inverse eigenvalue problems, SIAM J. Numer. Anal. 40, 2352-2367 (2003).

[31] F. Tisseur and K. Meerbergen, The quadratic eigenvalue problem, SIAM Rev. 43, 235-286 (2001).

[32] J.Y. Wang and B.S. Garbow, A numerical method for solving inverse real symmetric eigenvalue problems, SIAM J. Sci. Statist. Comput. 4, 45-51 (1983).

[33] S.-F. Xu, An Introduction to Inverse Algebraic Eigenvalue Problems, Peking University Press (1998).

[34] S. Zhou and H. Dai, The Algebraic Inverse Eigenvalue Problem (in Chinese), Henan Science and Technology Press (1991). 